\title{
Percepciones de papá y mamá sobre maestros varones en la educación inicial peruana
}

\author{
Parents' Perceptions on Male Teachers in Peruvian Preschool Education \\ Percepção do pai e da mãe sobre os professores do sexo masculino na educação inicial peruana
}

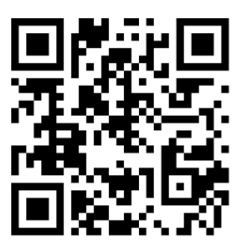

\section{Resumen:}

Objetivo. El presente artículo tuvo como finalidad explorar las percepciones de los padres y madres de familia con relación a la poca presencia de varones en el nivel inicial y a su ejercicio docente en estos espacios educativos. Metodología. Se empleó un enfoque de investigación cualitativo y de carácter exploratorio. Se realizó una entrevista semiestructurada a padres y madres de familia de centros educativos de nivel inicial públicos y privados de la ciudad de Lima (Perú). La información fue analizada de manera inductiva y clasificada en categorías que respondían al objetivo de la investigación. Resultados. Los principales resultados que emergieron de este proceso fueron: a) el rol de la sociedad en el ejercicio docente del nivel inicial; b) el dominio femenino y su rol maternal; c) desconfianza en la relación de varones y el cuidado de la niñez; y d) el varón como figura de disciplina, autoridad y juego. Discusión. Este estudio permite introducir diálogos en relación con el vínculo entre varón-infancia y abre una oportunidad para que las escuelas dirijan sus estrategias de cambio a fin de reducir la brecha de género masculina en este contexto docente. Asimismo, provee bases para desarrollar futuras investigaciones acerca del impacto de este grupo en los niños y niñas y en sus entornos próximos al conocer sus percepciones como actores educativos clave.

Palabras claves: Infancia; padres; percepción; rol; hombre, educación de la niñez. 
http://doi.org/10.15359/ree.25-3.33

http://www.una.ac.cr/educare

educare@una.ac.cr

\begin{abstract}
:
Objective. This article aimed to explore parents' perceptions concerning the low presence of men at the initial level and their teaching practice in these educational spaces. Method. A qualitative and exploratory research approach was used. A semi-structured interview was conducted with fathers and mothers of public and private initial-level educational centers in Lima (Peru). The information was analyzed inductively and classified into categories that responded to the objective of the investigation. Results. The main results that emerged from this process were the following: a) the role of society in the teaching exercise of the initial level; b) the female domain and her maternal role; c) distrust of the relationship between men and the care of young children; and d) the male as a figure of discipline, authority, and play. Discussion. This study introduces dialogues in relation to the link between male and childhood and opens an opportunity for schools to direct their change strategies in order to reduce the male gender gap in this teaching context. It also provides the basis to develop future research on the impact of this group on children and their immediate surroundings, knowing their perceptions as key educational actors.
\end{abstract}

Keywords: Childhood; parents; perception; role; man; childhood education.

\begin{abstract}
Resumo:
Objetivo. O objetivo deste artigo foi explorar as percepções de pais e mães em relação à baixa presença de homens no nível inicial e sua prática docente nesses espaços educacionais. Metodologia. Foi utilizada uma abordagem de pesquisa qualitativa e exploratória. Foi realizada uma entrevista semiestruturada com pais e mães de famílias de centros educacionais públicos e privados de nível inicial na cidade de Lima (Peru). As informações foram analisadas indutivamente e classificadas em categorias que respondiam ao objetivo da investigação. Resultados. Os principais resultados que emergiram desse processo foram: a) o papel da sociedade no exercício de ensino do nível inicial; b) o domínio feminino e seu papel materno; c) desconfiança na relação entre homens e cuidado de crianças; e d) o homem como figura de disciplina, autoridade e brincadeira. Discussão. Este estudo permite a introdução de diálogos em relação ao vínculo entre homem-infância e abre uma oportunidade para as escolas direcionarem suas estratégias de mudança, a fim de reduzir a diferença de gênero nesse contexto de ensino. Também fornece a base para o desenvolvimento de pesquisas futuras sobre o impacto desse grupo nas crianças e nos espaços imediatos, conhecendo suas percepções como atores educacionais essenciais.
\end{abstract}

Palavras-chave: Infância; pais; percepção; papel; homem; educação infantil.

\title{
Introducción
}

Desde las sociedades primitivas, comenta Zhang (2017), muchas de las tareas ejercidas eran divididas de acuerdo con el género de las personas de la comunidad. Esta división destinaba a las mujeres a ocuparse del cuidado, educación y alimentación de la tribu en especial de los más pequeños; mientras que los hombres ejercían labores de caza, fuerza y poder. Estos roles a causa del género han trascendido a lo largo de los tiempos y, sobre todo, en la educación de la primera infancia. Al respecto, Cunningham y Watson (2002) señalan que, si realizamos un vistazo rápido a un centro de cuidado infantil, preescolar o escuela primaria, se evidenciará la baja 
http://doi.org/10.15359/ree.25-3.33

representación de hombres que trabajan con niños pequeños, por lo que plantea reflexiones respecto a esta actividad. A estas reflexiones, Vendrell et al. (2015) añaden la percepción de las familias, las cuales se evidencian en la confianza que estas proporcionan a los docentes varones en su labor. Mencionan que para que los educadores tengan la confianza en los padres y las madres, y viceversa, necesitan alrededor de 3 meses para que la relación tome otro sentido. Asimismo, el estudio indica que el desarrollo de la desconfianza se puede ver influenciado por la variable vinculada a la creación de estereotipos que las familias muestran. Se encontró que $56,6 \%$ de las familias mantienen prejuicios respecto a los maestros varones y a su ejercicio docente en las edades de 0 a 3 años, mientras que el 27,7\% en el grupo etario de 3 a 6 años.

Por las razones expuestas, el propósito de la investigación se centra en explorar cuáles son las percepciones de padres y madres de familia respecto a la presencia docente masculina en las aulas de nivel inicial de la ciudad de Lima con el fin de recoger datos y experiencias de las familias cuando advierten que un varón podría estar a cargo de sus hijos o hijas más pequeños. Pretendemos así, contribuir a generar espacios de diálogo y reflexión respecto a los roles de género, la educación inicial y al impacto que se generan en los docentes varones y su actividad.

\section{Construcción social del género}

A diferencia del sexo, que se determina antes del nacimiento por los procesos biológicos, el género como proceso cultural y social crea diferencias sociales entre hombres y mujeres (Lorber, 1994). En su sentido más amplio es una construcción social e histórica elaborada sobre la base de las diferencias sexuales entre hombres y mujeres; y sus relaciones. Estas varían según la cultura, religión, economía, clases sociales, raza y momentos históricos, que afectan las acciones $y$, son influenciadas por relaciones de poder que revelan conflictos y contradicciones que caracterizan a la sociedad (Butler, 2005).

La construcción social del género se convierte en una de las formas en las que el ser humano ha organizado su vida. Las sociedades dependen de divisiones o de la formación de grupos de personas para el cumplimiento de ciertas tareas. Una de las maneras de organización se realiza en base al género, la raza o la etnia (Lorber, 1994). Desde un punto individual, la construcción social de género empieza desde que se le asigna el sexo a un bebé en base a los genitales que presenta; para que posteriormente, se le vista o adorne de la manera que socialmente se cree que un género debe verse (Lorber, 1994). Luego, cuando el género del niño o niña se vuelve evidente, la sociedad ofrece tratos diferenciados, a los que el niño responde sintiéndose y actuando diferente (West y Zimmerman, 1987). En este sentido, los estereotipos de género se presentan como creencias sobre las características, atributos y comportamientos de los miembros de cierto grupo (Wood, 2008). El aspecto cognitivo de la actitud de la persona en un grupo puede definir cómo son los atributos que él o ella asigna en sus relaciones. Por ejemplo, la creencia de que los hombres son agresivos se debe a la asociación que este ha imputado en los grupos sociales, ya sea de forma positiva o negativa (Eagly y Mladinic, 1989). 
http://doi.org/10.15359/ree.25-3.33

http://www.una.ac.cr/educare

educare@una.ac.cr

\section{El rol masculino y el ámbito laboral}

Las características biológicas de un grupo, determinan ciertos comportamientos sociales, en donde los patrones de género asociados a la sexualidad, paternidad, trabajo y costumbres en niños, adolescentes y adultos se ven reflejados en sus interacciones y actuaciones en un determinado contexto (Lorber, 1994; Pizarro Quiñones, 2018). Es así como, de forma tradicional, se ha normalizado que la maternidad -y especialmente la responsabilidad del cuidado del niño- coexiste en un solo género: el femenino. Tal rol se ha asociado a la capacidad biológica de procrear asumiendo con ello, un tácito comportamiento social (Pizarro Quiñones, 2018). Del mismo modo sucede con los varones, donde su corporalidad -entendida como apta para el desarrollo exclusivo de ciertas actividades- se convierte en la justificación para la división del trabajo, presencia en ciertos dominios o formas de desarrollo de una actividad (Haslanger, 2012).

De acuerdo a Faur (2004), el concepto de masculinidad existe en la medida que lo haga también el de feminidad, bajo el sentido de lo opuesto o complementario. Ambas definiciones parten del hecho de reconocer la diferencia. Son conceptos relacionales, pues suponen ciertas representaciones y desarrollos atribuibles a lo masculino y lo femenino. Estas representaciones no solo son distintas entre sí, sino que no tendrían sentido sin su contra-cara (Connell, 2005). Por ejemplo, la actividad, la razón e independencia son características propias de un hombre y su masculinidad, en cambio la pasividad, emoción y dependencia corresponden a una mujer y su feminidad (Davies, 1989). Sin embargo, los hombres no son todos homogéneos. Esto se refiere a la manera en que presentan o desenvuelven su género. Incluso, esta presentación es afectada por las demandas del contexto inmediato y el largo registro de género en las sociedades (Sargent, 2005). Asimismo, se habla de la masculinidad y la feminidad como normas de género que se asocian a los roles de género. Estas prácticas, en especial la masculinidad hegemónica, son aquellas que naturalizan o normalizan la dominancia masculina y la subordinación femenina (Haslanger, 2012). Hombres, afeminados, de orientación homosexual e incluso aquellos heterosexuales que cuidan de niños o niñas y transgreden tal definición tradicional son frecuentemente vulnerados y tienden a ser ridiculizados por otros hombres que reprueban o desafían esta hegemonía, y a su vez son catalogados como traidores a la masculinidad pudiendo vivir violencia psicológica o emocional (Sargent, 2005).

A este desarrollo se le añade que el rol masculino en la sociedad ha sido vinculado directamente con la idea de jefe de hogar, idea contraria al rol que se le ha atribuido a la mujer como la reina de la casa. Esta construcción coloca tanto a los varones como a las mujeres frente a ciertas demandas sociales que deben asumir. Los varones, por ejemplo, se ubican dentro del rol protector, de poder, de dominación, y de proveedores (Sánchez Barrenechea, 2011). $Y$, justamente para cumplir con este último, el hombre tiene la responsabilidad de salir de su espacio privado al espacio público para trabajar (Cáceres et al., 2002). 
http://doi.org/10.15359/ree.25-3.33

En el contexto de la industrialización, el rol masculino cobró mucha importancia, ya que las fábricas solían contratar únicamente a los varones. Entonces, se empezó a identificar con mayor fuerza al hombre como trabajador dentro del espacio público, mientras que, a la mujer, dentro del privado. Se empieza a entender la función del varón como responsable del trabajo asalariado y la de la mujer como garante de las tareas domésticas y del hogar; entre ellas, la del cuidado de los niños y niñas (Valdivia Santa Cruz, 2013). Aun así, la diferenciación del rol de la mujer como mundo privado-no trabajo y la del hombre como público-trabajo, en la actualidad, ya no se encuentra muy marcada debido a que la falta de trabajo genera que el rol social masculino de proveer se encuentre muchas veces limitado, otorgándole a la mujer un nuevo estatus social como proveedora (Cáceres et al., 2002). Sin embargo, es preciso recalcar según Lomazzi et al. (2019) que numerosos estudios muestran que, si bien las mujeres han ingresado al mundo laboral a realizar trabajos que tradicionalmente se ha designado a la fuerza masculina, el proceso continúa siendo lento.

\section{Maestros en la educación inicial}

La desigualdad de género y los roles sociales también han impactado en el contexto educativo. Por un lado, desde el inicio, la educación escolar ha estado destinada al cuidado y a la alimentación de los niños y las niñas. Por ello se ha considerado pertinente, por su rol construido socialmente, designar esta función a las mujeres, lo cual provoca un desequilibrio de género en la enseñanza, especialmente en la educación inicial (Zhang, 2017). Este imaginario se ha plantado con mayor fuerza en la sociedad, generando que se hable de las escuelas como espacios femeninos. Esta definición está más presente en contextos como guarderías o de los primeros grados escolares. En oposición, se califica a la escuela como espacio masculino solo cuando tiene fines de (re)producción de conocimientos científicos, proceso que históricamente se ha asignado a hombres y ha estado bajo su dominio (Cappi Manzini y do Horto Salles Tiellet, 2014).

Los trabajos y las profesiones se han delimitado y construido socialmente de acuerdo con el sexo; designando a la docencia como una opción de profesión exclusivamente femenina, especialmente durante los primeros años (Pico Villancís, 2017). Esta determinación es recogida por algunos padres y madres que poseen una visión basada en prejuicios en relación con los hombres que ejercen la labor de educador en la primera infancia. Entienden que este es un trabajo exclusivamente femenino por estar relacionado con el amor, cuidado y afecto, deteriorando el prestigio del hombre en la educación preescolar. Lo mismo sostiene García Villanueva y Hernández Ramírez (2015) mencionando que la posibilidad de que los hombres ejerzan la labor de educador en el preescolar es mínima, debido a que los padres y las madres consideran que es una profesión únicamente femenina, centrada en la protección, servicio, además permite dar continuidad a la maternidad, lo cual garantiza el amor incondicional.

Como mencionan Joseph y Wright (2016) debido a la presión social, a los prejuicios de los padres y a la limitada oportunidad laboral de los docentes masculinos en la educación inicial, la presencia de 
http://doi.org/10.15359/ree.25-3.33

http://www.una.ac.cr/educare

educare@una.ac.cr

maestros varones es casi inexistente. En algunos estados de Australia, la ley no permite que varones ejerzan la labor de cuidadores familiares, los cual se demuestra en las estadísticas, donde alrededor del $2 \%$ de los varones conforman la profesión de la primera infancia (Clyde, 1989). Del mismo modo, en Nueva Zelanda la realidad no es diferente, ya que menos del 1\% de los docentes de la primera infancia son hombres. Esto se debe a los prejuicios públicos generalizados de que las mujeres solo pueden cumplir los roles de cuidados, sirviendo como obstáculo para los hombres al contemplar carreras en la educación temprana (Joseph y Wright, 2016). En América Latina la situación es similar. En el caso de Chile, en la educación del nivel inicial, la baja representatividad masculina es notable, ya que el $99,5 \%$ de la fuerza laboral está conformada por mujeres, donde solo existe un $0.5 \%$ de docentes varones según la Organización de las Naciones Unidas para la Educación, la Ciencia y la Cultura (Pardo y Adlerstein, 2015). Del mismo modo, en el caso de Colombia se evidencia diferencias claras en cuanto al porcentaje de docentes varones, donde solo existe un $30 \%$ de hombres en la educación inicial, el cual es bajo frente al de las mujeres (Muñoz Restrepo y Vázquez Holguín, 2017). En el contexto peruano, este problema se visualiza en tanto el $7 \%$ de las personas docentes de nivel inicial son de género masculino y el 37\% en el primer y segundo grado de educación primaria según un estudio realizado por el Instituto Nacional de Estadística e Informática (INEl, 2017).

Los prejuicios van en la línea de la preocupación de los padres y las madres de familia al creer que los docentes varones son más propensos a abusar de los niños. Estas suposiciones permiten el alejamiento de los docentes varones de la enseñanza en el preescolar, haciendo que pocos hombres elijan dicha profesión (Joseph y Wright, 2016). De la misma manera, las suposiciones también vienen al crear construcciones particulares en torno de la homosexualidad y la pedofilia. Estos son términos muchas veces cargados emocionalmente, ya que pueden actuar como un mecanismo de control social para mantener un limitado número de maestros varones en este nivel (Zhang, 2017).

Las mencionadas construcciones sociales son rutas de acceso a la creación de la estructura de género en la educación inicial, ya que la imagen y el cuidado de los niños y las niñas como trabajo exclusivo de mujeres está respaldada por el instinto de madre y maternidad en la creación de puestos y las funciones de trabajo dentro del preescolar. Sin embargo, ante todo lo expuesto, es importante mencionar también que la presencia del hombre en la educación inicial genera otras interpretaciones del modelo masculino que se encuentran en conflicto con los mencionados prejuicios. La primera, es la creencia de que los maestros pueden actuar como padres sustitutos. Del mismo modo, proporcionarán un modelo de masculinidad al convivir con los niños y las niñas, contribuyendo a un aumento de la disciplina y a la disminución de los problemas de comportamiento (Sargent, 2005). Diversas instituciones infantiles de preescolar tienden a limitar la labor de los hombres como docentes; asignándoles roles administrativos en equipos directivos. En este sentido, se ha proporcionado una limitada atención a los hombres que han optado por la docencia en la educación preescolar condicionándolos a menores oportunidades laborales por la inseguridad mencionada que transmiten a las familias (Pico Villancís, 2017).

6

Los artículos de la Revista Electrónica Educare del Centro de Investigación y Docencia en Educación de la Universidad Nacional, Costa Rica, se comparten bajo términos de la Licencia Creative Commons: Reconocimiento, № Comercial, Sin Obra Derivada 3.0 Costa Rica. Las autorizaciones adicionales a las aquí delimitadas se pueden obtener en el correo: educare@una.cr 


\section{Metodología}

La metodología empleada en la investigación responde a un enfoque cualitativo y tiene la finalidad de recopilar datos sobre un problema para analizarlos de forma inductiva. Este enfoque permite observar el fenómeno de cerca en un entorno natural, en un contexto determinado y con un grupo de personas escogidas (Creswell y Poth, 2018). El hacerse cargo de una investigación cualitativa supone y requiere un fuerte compromiso para estudiar el problema con la idea de que la demanda de tiempo y recursos será alta (Creswell y Poth, 2018). Asimismo, se debe tener en cuenta que el tema de investigación se abre a la posibilidad de distintas perspectivas y puntos de vista dependiendo del participante entrevistado, razón por la cual se exige un esfuerzo por mirar con cierta distancia, reflexionar y poder confrontar las ideas propias de las personas investigadoras y tomarlas en cuenta para hacer más enriquecedor el proceso y desarrollo del estudio (Osses Bustingorry et al., 2006).

La investigación tuvo un carácter exploratorio con influencia de la teoría fundamentada. Esta aproximación es un método de análisis comparativo que busca desarrollar conceptos integrados con el fin de brindar una predicción, explicación teórica, interpretación y aplicación de algún fenómeno social (Corbin y Strauss, 1990). Del mismo modo, nos permite identificar categorías teóricas mediante la comparación de los datos cualitativos obtenidos (Creswell y Poth, 2018; Páramo Morales, 2015). El énfasis recae en la relevancia del proceso de codificación que se efectúa de estos datos con el objetivo de identificar categorías, las cuales pueden contener subcategorías agrupadas en dimensiones con propiedades comunes. En consecuencia, mediante un análisis inductivo, se permite la construcción de una teoría sobre el objeto de estudio (Corbin y Strauss, 1990).

Este análisis inductivo enriquece la investigación en la medida que permite ahondar en las concepciones y opiniones que no se visibilizan, pero que existen en cada una de las relaciones y en el accionar del grupo estudiado. Siguiendo este proceso es posible desarrollar líneas de investigación emergentes que -a partir del tema central-encuentren nuevos cuestionamientos sobre los maestros varones frente a otros agentes involucrados en el contexto educativo.

\section{Participantes}

Las personas participantes en este estudio fueron padres y madres de niños del nivel inicial de varios centros educativos públicos y privados de Lima (Perú). Este nivel pertenece a la educación básica regular peruana, atendiendo edades desde 0 a 6 años. Se tomó en cuenta que al menos la mitad tuvieran hijos o hijas a cargo de un docente varón. Esto significó una complicación para el estudio, pues no es frecuente encontrar docentes varones en el nivel inicial (0 a 6 años) debido a la baja representación masculina en este contexto particular. Se pensó, desde el inicio, apostar por una participación equitativa entre padres y madres de familia. Sin embargo, durante el proceso, se presentaron cambios asociados a la accesibilidad y disponibilidad de cada participante, en especial a la de los padres de familia. En tal sentido, fue 
http://doi.org/10.15359/ree.25-3.33

http://www.una.ac.cr/educare

educare@una.ac.cr

mayor la presencia de madres dispuestas a formar parte del grupo de estudio y con el tiempo necesario para el desarrollo de las entrevistas. La Tabla 1 presenta, a continuación, algunos alcances generales sobre las personas entrevistadas.

Tabla 1: Alcances sobre los padres y madres entrevistados

\begin{tabular}{|c|c|c|c|c|}
\hline Participante & $\begin{array}{l}\text { Género } \\
\text { (M/F) }\end{array}$ & $\begin{array}{l}\text { Sector educativo de } \\
\text { su(s) hijo/a(s) }\end{array}$ & $\begin{array}{l}\text { Edad del hijo/a } \\
\text { (años) }\end{array}$ & $\begin{array}{c}\text { Género del hijo/a } \\
\text { (M/F) }\end{array}$ \\
\hline $\mathrm{N}^{\circ} 1$ & $\mathrm{~F}$ & Privado & 4 y 4 & F y $M$ \\
\hline $\mathrm{N}^{\circ} 2$ & $\mathrm{~F}$ & Privado & 5 & $\mathrm{~F}$ \\
\hline$N^{\circ} 3$ & $M$ & Privado & 5 & $\mathrm{~F}$ \\
\hline$N^{\circ} 4$ & $\mathrm{~F}$ & Privado & 5 & M \\
\hline$N^{\circ} 5$ & $\mathrm{~F}$ & Privado & 5 & $M$ \\
\hline $\mathrm{N}^{\circ} 6$ & $\mathrm{~F}$ & Privado & 6 & M \\
\hline$N^{\circ} 7$ & $\mathrm{~F}$ & Público & 5 & $\mathrm{~F}$ \\
\hline$N^{\circ} 8$ & $\mathrm{~F}$ & Público & 3 & $\mathrm{~F}$ \\
\hline$N^{\circ} 9$ & $\mathrm{~F}$ & Público & 3 & $\mathrm{~F}$ \\
\hline$N^{\circ} 10$ & $F$ & Público & 3 & $\mathrm{~F}$ \\
\hline $\mathrm{N}^{\circ} 11$ & $\mathrm{~F}$ & Público & 3 & $\mathrm{~F}$ \\
\hline $\mathrm{N}^{\circ} 12$ & $\mathrm{~F}$ & Público & 3 & $M$ \\
\hline$N^{\circ} 13$ & $\mathrm{~F}$ & Privado & 4 y 4 & Fy $M$ \\
\hline $\mathrm{N}^{\circ} 14$ & $M$ & Privado & 4 & $\mathrm{~F}$ \\
\hline$N^{\circ} 15$ & $M$ & Privado & 4 & $M$ \\
\hline $\mathrm{N}^{\circ} 16$ & $\mathrm{~F}$ & Público & 4 & $M$ \\
\hline $\mathrm{N}^{\circ} 17$ & $\mathrm{~F}$ & Privado & 3 & $\mathrm{~F}$ \\
\hline $\mathrm{N}^{\circ} 18$ & M & Privado & 3 & $\mathrm{~F}$ \\
\hline$N^{\circ} 19$ & $\mathrm{~F}$ & Público & 5 & $\mathrm{~F}$ \\
\hline
\end{tabular}

Nota: Elaboración propia. 
http://doi.org/10.15359/ree.25-3.33

\section{Recolección de información}

Las entrevistas semiestructuradas proporcionan mayor flexibilidad sin perder el foco de la conversación y tiene la capacidad de develar facetas escondidas del individuo (Qu y Dumay, 2011). Tales entrevistas se realizaron de manera presencial y en algunos casos por vía telefónica siguiendo tres ejes temáticos: (1) estereotipos de género; (2) brecha de género en la docencia inicial y (3) roles de género en la docencia de la educación inicial (VerTabla 2). Durante el proceso, se recordó a las personas participantes el propósito del estudio otorgándoles la oportunidad de llenar un consentimiento informado. Asimismo, se les solicitó el permiso correspondiente para realizar la grabación digital de la entrevista enfatizando la confidencialidad de sus respuestas con la finalidad de asegurar los procedimientos éticos previstos en el proceso de investigación. Para brindarles mayor seguridad y confianza a las personas participantes respecto al uso de la información obtenida, se solicitó también un compromiso a la institución asegurando que las opiniones no comprometerían el vínculo de las personas participantes ni el de las personas menores de edad; por ser absolutamente confidenciales y para fines estrictamente académicos.

Tabla 2: Ejes temáticos de la entrevista

\begin{tabular}{ll}
\hline \multicolumn{1}{c}{ Eje temático } & \multicolumn{1}{c}{ Alcances } \\
\hline Estereotipos de género & $\begin{array}{l}\text { Aborda las creencias sobre las características, atributos y } \\
\text { comportamientos de los miembros de cierto grupo, en este caso de } \\
\text { una mujer y de un varón establecido por la sociedad. }\end{array}$ \\
Brecha de género en la docencia inicial & $\begin{array}{l}\text { Aborda la desigualdad en la inserción y el desempeño del docente del } \\
\text { nivel inicial en base su género (femenino o masculino). }\end{array}$ \\
$\begin{array}{l}\text { Roles de género en la docencia de la } \\
\text { educación inicial }\end{array}$ & $\begin{array}{l}\text { Aborda la idea del rol maternal de la mujer, lo cual destina a la } \\
\text { educación inicial como su dominio a comparación del rol proveedor, } \\
\text { de dominación y de protección del varón, incapaz de ejercer cuidado a } \\
\text { niños y niñas pequeños. }\end{array}$ \\
\hline
\end{tabular}

Nota: Elaboración propia.

\section{Resultados y discusión}

A partir del proceso de codificación de las entrevistas fue posible explorar y contrastar la percepción de las madres y los padres de familia sobre la baja representación masculina en la educación inicial. Las categorías obtenidas de manera inductiva se pueden apreciar en la siguiente codificación abierta presentada en la Figura 1. De ellas, las sombreadas constituyen las que tuvieron mayor recurrencia. 
http://doi.org/10.15359/ree.25-3.33

http://www.una.ac.cr/educare

educare@una.ac.cr

Figura 1: Categorías frecuentes en la primera codificación

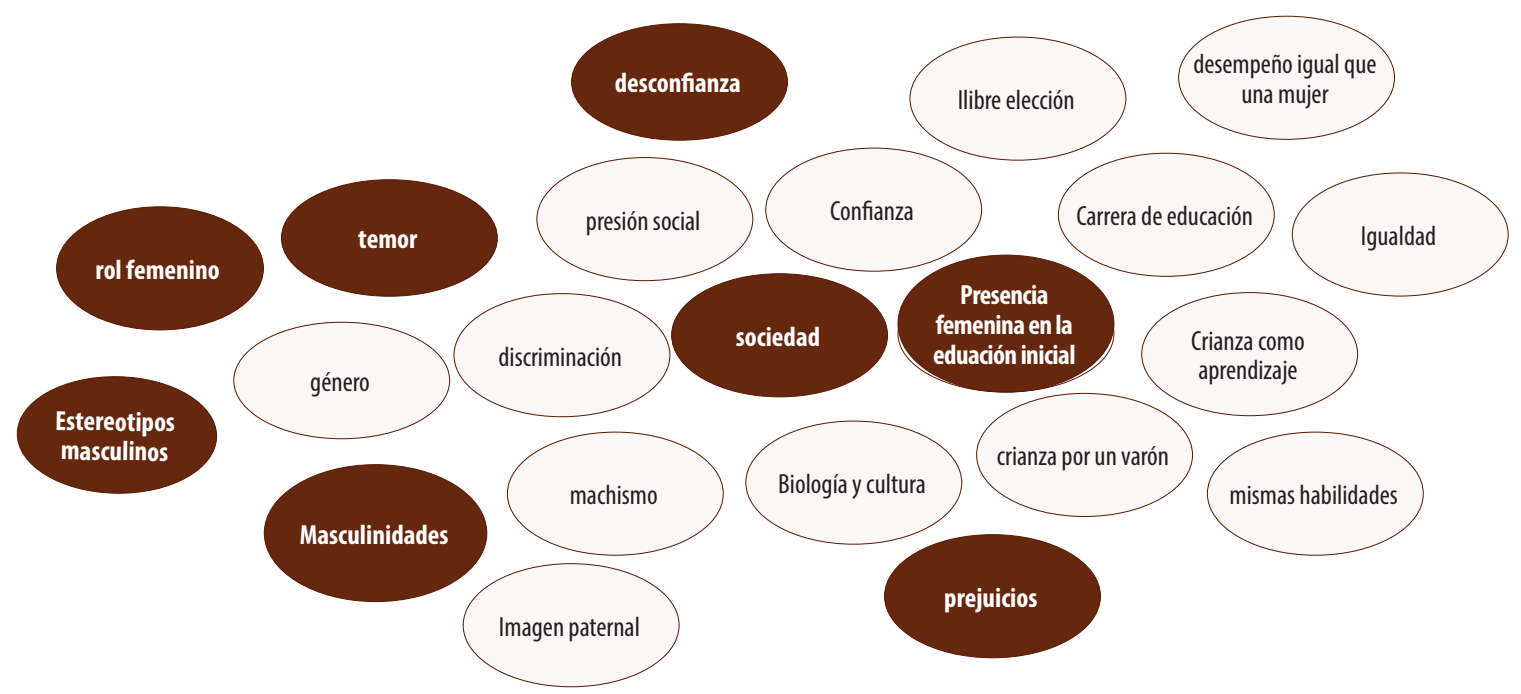

Nota: Elaboración propia.

Las etiquetas más recurrentes que se presentaron fueron: rol femenino, temor, la desconfianza, los prejuicios, las masculinidades, los estereotipos masculinos, la presencia femenina en la educación inicial y la sociedad. Estas están asociadas a otras categorías de menor recurrencia que se vinculan entre sí por medio de relaciones que permitieron identificar otros conceptos generales. En una segunda codificación, las categorías anteriores fueron a su vez agrupadas en categorías que engloban algunos conceptos, las cuales se muestran en la Figura 2.

Siguiendo la metodología planteada, se realizó una tercera y última codificación que facilitó la determinación de conceptos más generales. En consecuencia, los temas centrales de análisis obtenidos fueron los siguientes: a) el rol de la sociedad en el ejercicio docente del nivel inicial; b) dominio femenino y su rol maternal en el nivel inicial c) desconfianza en varones respecto a la crianza/cuidado de niños pequeños; d) el varón como figura de disciplina, autoridad y juego. 
Figura 2: Categorías frecuentes de la segunda codificación

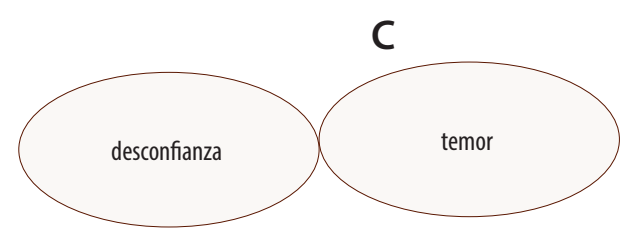

A
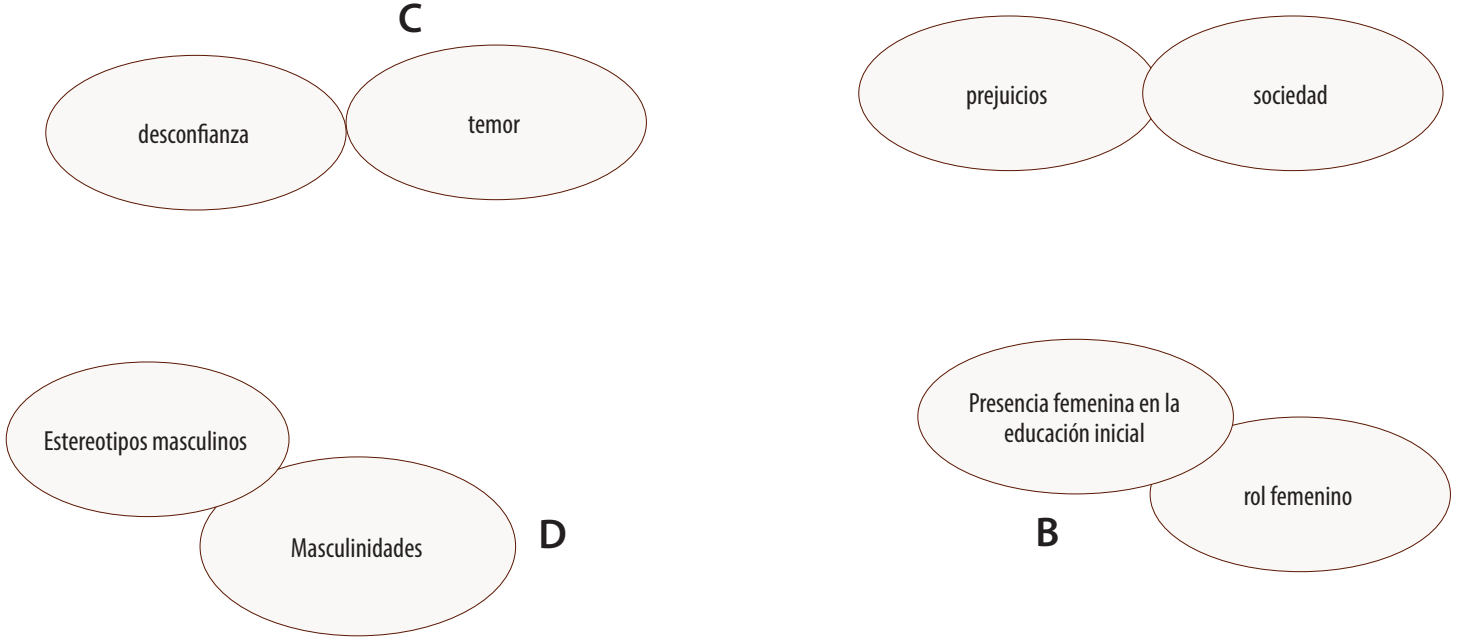

Nota: Elaboración propia.

\section{a) El rol de la sociedad en el ejercicio docente del nivel inicial}

Padres y madres de familia opinan que el ejercicio docente en el nivel inicial por parte de los varones se ve influenciado por la sociedad y por cómo esta sostiene prejuicios sobre la masculinidad ligados a la profesión docente. En primer lugar, mencionan que existe cierta presión social para que los varones no elijan la carrera de educación como una opción, y en especial la del nivel inicial. Esto por el temor al juicio social externo.

[Prefiero una maestra] porque en la sociedad está mal visto que un hombre sea docente de inicial. (Participante 9)

Como se puede ver en estos testimonios, padres y madres percibieron que son los estereotipos y los prejuicios hacia los varones los que han generado que ellos se sientan apartados de estos espacios educativos. La sociedad es, para ellos, quien destina al varón características de rudeza que incluso inspiran desconfianza e inseguridad.

[Prefiero una maestra] porque casi el 99\% de los abusadores son hombres (Participante 4).

[Siento desconfianza] por el temor de que son hombres y por todo lo que pasa. Por los tocamientos y la falta de delicadeza. (Participante 10) 
http://doi.org/10.15359/ree.25-3.33

http://www.una.ac.cr/educare

educare@una.ac.cr

Asimismo, los padres y madres mencionaron que, parte de los prejuicios se ven influenciados por las noticias, estadísticas e incluso en la publicidad. En esta refieren al varón como actor de tocamientos y abusos, en su mayoría sexual, de niños y niñas; y a la mujer, como imagen principal de la educación inicial.

[Me preocupa tener un docente masculino en inicial] por el mismo contexto, pues vivimos en una sociedad machista. (Participante 12)

[Prefiero a una maestra] porque [los niños y las niñas] son más pequeños y tienen cierta confianza más con una mujery por lo mismo que [el Perú] es un país machista. (Participante 13)

Por último, muchas de las percepciones exploradas de las entrevistas identificaron a la sociedad peruana como machista. Mencionan que la presencia de un varón en aulas de inicial se debe a las actitudes y contextos de machismo, lo cual refuerza la confianza en una mujer por el rol de cuidadora que se le ha otorgado.

En esta línea, algunos actores sostienen que tradicionalmente, el rol principal que ha asumido la mujer está relacionado al cuidado y la capacidad de procrear (Pizarro Quiñones, 2018). Por el contrario, los hombres asumen roles de protección, de poder, dominación y como proveedores o jefes del hogar (Sánchez Barrenechea, 2011). Así, el rol de la sociedad y la construcción que esta ha proporcionado impacta directamente en las acciones que cada persona asume en su interacción con los otros, impidiéndoles desenvolverse con libertad e igualdad. Del mismo modo, los roles sociales también han impactado en aspectos educativos, ya que existe la presión social, motivo por el cual los hombres son ridiculizados por otros cuando se encargan del cuidado de los niños y las niñas (Sargent, 2005). Además, muchos de los prejuicios se ven relacionados a la preocupación de los padres y madres de familia al imaginar que un docente varón puede estar más expuesto a abusar de los niños y las niñas (Joseph y Wright, 2016). La sociedad cumple un rol importante en cuanto a las concepciones o pensamientos que cada persona pueda asumir en relación la presencia masculina en inicial. Estas construcciones y roles que se han designado a un varón y a una mujer funcionan como obstáculos para que los hombres contemplen carreras vinculadas al contacto con niños o niñas.

\section{b) Dominio femenino y su rol maternal en la educación inicial}

El grupo de estudio coincidió que la presencia de la mujer en la educación de nivel inicial es muy recurrente. En primer lugar, muchos de los entrevistados concuerdan en que son las mujeres quienes postulan a carreras de educación, sobre todo al nivel inicial, a comparación de los varones. Esto debido a su estrecha vinculación con el rol maternal que tienen con los niños y niñas más pequeños y a su acogida con este grupo etario. 
http://doi.org/10.15359/ree.25-3.33

[Es normal la presencia de una maestra en inicial] porque la población [de mujeres] es mayor y porque son mamás o tienen mayor acogida con los niños- (Participante 12)

Es bastante notorio [que haya más mujeres como maestras de inicial] pues es parte de la elección profesional por parte de las mujeres. (Participante 17)

Del mismo modo, estas percepciones refuerzan la vinculación de mujer con la educación de niños pequeños, pues mencionan que se asume la continuidad del hogar, del rol maternal y de cuidado que las mujeres ejercen en su entorno.

[Las maestras de inicial son en su mayoría mujeres] porque hay una continuidad que se asume al cuidado de la madre (Participante 6)

[Prefiero una maestra en inicial] porque puede atender mejor a los niños, ya que buscan más la figura materna. (Participante 11)

El rol maternal se evidencia en las entrevistas como asunto relevante para ser maestra de inicial por las características que de ella se demanda. Mencionaron que, entre una mujer y un niño o niña, existe una conexión biológica y distinta al de un varón al llevarlo dentro de su cuerpo por un largo período hasta su nacimiento. Además, refuerzan que una madre puede manipular de manera más cuidadosa el cuerpo de un recién nacido.

Estos testimonios fortalecen la hipótesis de algunos actores al señalar que los padres y madres de familia consideran a la educación inicial como una profesión exclusivamente femenina, ya que está relacionada con el servicio, cuidado y protección. Todo ello vinculado a la continuación de la maternidad (García Villanueva y Hernández Ramírez, 2015). Por este motivo, la presencia masculina es mínima. En el contexto peruano, según el INEI (2017) la presencia masculina en inicial es de 7\%. En este punto, los resultados del estudio parecen confirmar lo planteado por estos especialistas, pues los sujetos entrevistados anteponen la presencia femenina en inicial, ya que esta se relaciona al amor incondicional y al cuidado.

\section{c) Desconfianza en la relación de varones y crianza/cuidado de la niñez pequeña}

Los padres y madres entrevistados perciben como otro tema relevante la relación entre el varón y niño pequeño, en especial, de su cuidado o crianza. Para empezar, muchos de los comentarios rescatan este ejercicio como proceso de aprendizaje, es decir, solo se puede realizar si es que se tiene la formación o conocimientos necesarios sobre este tema (paternidad) e incluso si es que se es padre. 
http://doi.org/10.15359/ree.25-3.33

http://www.una.ac.cr/educare

educare@una.ac.cr

[Un varón podría asumir el cuidado de un niño] sólo si es [una persona] conocida, como el padre, porque no va a venir alguien extraño para llevarla a los servicios higiénicos. (Participante 7)

Asimismo, es preciso recalcar que las percepciones de las personas entrevistadas señalan que, en la actualidad, el varón está tomando mayor protagonismo en estos espacios de crianza, pues los niños y las niñas desarrollan un mejor apego. Sin embargo, algunos describen que este tipo de crianza dependerá de las experiencias familiares o de otros entornos próximos que ha tenido el cuidador.

[La mujer y el varón pueden dedicarse al cuidado de un niño] porque en mi casa me enseñaron que no existen princesas ni príncipes. (Participante 10)

[Ambos pueden desempeñar el rol de cuidador] porque hay padres y varones muy buenos, [es que] todo viene de casa. (Participante 13)

Este aspecto es particularmente importante, pues deja claro que el rol imaginario que se les ha designado a los varones está dando un giro en cuanto a las posibilidades de cuidado/ crianza que un varón puede ejercer, al igual que una mujer. Es importante mencionar que este giro fue mencionado en su mayoría por las madres y padres que pertenecen a centros privados e incluso añaden, que no consideran relevante que un varón esté a cargo del aula de sus hijos o hijas pequeños. En cambio, la mayoría de entrevistados de colegios públicos si demostró temor y desconfianza al plantearle la misma premisa, agregando que el rol de cuidador dependerá de las vivencias que ha obtenido dentro de su familia.

En base a estos resultados obtenidos en el estudio, no se encontró información que fundamente y que esté relacionada a la desconfianza del varón en la crianza/cuidado de niños pequeños. Por ello, convendría seguir explorando en el contexto estudiado a este tipo de aceptación del docente varón en correlación con el ejercicio de su propia paternidad.

\section{d) El varón como figura de disciplina, autoridad y juego}

Los padres y madres de familia perciben que la presencia de un varón como docente del nivel inicial podría ser ventajoso cuando se desarrolla un modelo de masculinidad que se vincula a la practicidad, energía y firmeza.

El docente varón es [más] enérgico, en cambio las maestras de inicial son dulces. (Participante 8)

[Un varón como docente de inicial impactaría] en la firmeza; en hacer que los niños hagan caso a las indicaciones. (Participante 14) 
http://doi.org/10.15359/ree.25-3.33

A este modelo de masculinidad se le añade la figura paterna que ejercería el varón siendo docente de niños pequeños. Es importante para las personas entrevistadas que los niños y las niñas tengan una imagen representativa paternal que demuestra el cumplimiento de reglas y que mantenga el orden.

Los padres siempre ponen sus reglas [entonces] la figura paterna siempre es buena para que ponga un poco más de orden. (Participante 7)

Adicionalmente, los entrevistados y las entrevistadas mencionaron que tener la presencia de un varón en el nivel inicial podría favorecer a los niños y a las niñas en cuanto al desarrollo de su autoestima, pues la figura paterna ejercida les garantiza sentimientos de seguridad y protección.

[Un maestro de inicial puede generar] seguridad y protección a los niños y a las niñas. (Participante 20)

Del mismo modo, algunos de las personas entrevistadas comentaron que un docente en las aulas de inicial incentivaría que niños y niñas tomen mayor riesgo para realizar sus actividades o juegos y al mismo tiempo, que las niñas puedan desenvolverse en deportes como el fútbol.

El mismo hecho social [dispone que] un docente varón anime a los niños a realizar cosas más arriesgadas; como también una docente mujer, permita desarrollar formas más cuidadosas de relación con el otro. (Participante 2)

La ventaja sería [que un varón tendría] más alegre al grupo. Tal vez las niñas jugarían más fútbol. (Participante 8)

Estos resultados, se contrastan con lo que sostiene Connell (2005) al mencionar que la masculinidad y la feminidad pueden desarrollarse como complemento u oposición. El ejercicio de la masculinidad de un docente varón supone determinadas prácticas de firmeza y riesgo. Asimismo, tal como señala Davies (1989) la actividad o juego, la razón e independencia son características propias de un hombre y su masculinidad, en cambio la pasividad, las relaciones cuidadosas y emoción corresponden a una mujer y su feminidad. Por otro lado, la idea de figura paterna que da seguridad y fortalece la autoestima se vincula con lo que menciona Sargent (2005), que, al tener un docente varón en inicial, se asume el rol de padre sustituto. En ese sentido, la presencia masculina puede influir como figura de disciplina y autoridad, ya que estos pueden contar con la capacidad de hacer cumplir las reglas y mantener el orden.

Es importante resaltar que convendría seguir una línea de investigación en relación con el impacto de un varón en los niños y las niñas de la educación inicial y si estas contrastan con la opinión de las familias. Asimismo, sería útil explorar las percepciones de aquellos actores a quienes se les imparte educación: los niños y niñas. Conocer sus opiniones, posturas o ideas acerca de su docente reformularía propuestas e intervenciones desde las instancias pertinentes. 
http://doi.org/10.15359/ree.25-3.33

http://www.una.ac.cr/educare

educare@una.ac.cr

\section{Conclusiones}

Un docente varón en inicial es señalado, en mayor medida, desde su rol y la connotación negativa que se le ha otorgado a su género a raíz de las noticias y estadísticas. En este sentido, para la mayoría de los padres y madres de familia poco de relevancia tiene la preparación y profesionalismo de un varón ejerciendo su rol como docente de la primera infancia, lo cual refuerza aquellos estereotipos vinculados a su desempeño en este espacio.

Los entrevistados y las entrevistadas mencionaron que la crianza o cuidado hacia niños pequeños por parte de un varón solo se debe realizar en espacios o entornos de confianza. Esto nos lleva a reflexionar si acaso las escuelas no son espacios de confianza para los padres y madres de familia, por lo que no dejarían a su hijo o hija bajo el cuidado de docentes de este género. Las escuelas debieran repensar acerca de cuál imagen y cuáles estrategias están facilitando a las familias para que se sientan convencidos y confiados de optar por su institución.

Por su parte, existe una preocupación mayor por parte de las personas entrevistadas con relación al cuerpo y la sexualidad de sus hijos e hijas. Es importante mencionar que las percepciones se vinculan en su mayoría a la manipulación transgredida de los cuerpos de los niños y niñas, más allá de aspectos emocionales, cognitivos o sociales. Este punto nos invita a pensar en estrategias de defensa o seguridad que niños y niñas debieran desarrollar para dar a conocer su voz ante situaciones de abuso teniendo al adulto de aliado y persona en quien confiar.

Resultó revelador encontrar diferencias entre las opiniones de los padres y madres de familia cuyos hijos e hijas se encuentran en un colegio privado frente a las familias de colegios públicos. Bajo el entendido de que los colegios privados, en el contexto estudiado, concentran mayor población perteneciente a las clases más privilegiadas y los colegios públicos a familias con menores recursos económicos; resulta interesante seguir ahondando en torno a la mayor aceptación en cuanto a la presencia de un varón en las aulas del nivel inicial en las instituciones privadas y a la confianza que existe sobre ellos. Este hallazgo invita a seguir explorando en estudios posteriores en torno a aquellos factores que forman y consolidan tales opiniones y representaciones de cada familia respecto al nivel de aceptación del género del docente en correlación a sus propios contextos socioeconómico y cultural.

Finalmente, a pesar de que en ciertos sectores de la población se normaliza más el que profesores varones estén a cargo de estudiantes de preescolar, los argumentos que las familias esgrimen al afirmar que el cuidado y extensión de lo maternal es indisociable del género femenino -en contraposición a la toma de riesgo y sentido de orden o firmeza ejercida por el género masculino- siguen reforzando los clásicos estereotipos de género y las construcciones hegemónicas en torno al ser hombre y ser mujer; y a los atributos dados a cada uno en el entorno en el que se desenvuelven. Esto constituye una tarea aún pendiente de seguir trabajándose desde la mirada educativa y muy especialmente desde la primera infancia. 


\section{Declaración de Material complementario}

Este artículo tiene disponible, como material complementario:

-La versión preprint del artículo en https://doi.org/10.5281/zenodo.4890849

\section{Referencias}

Butler, J. (2005). Gender trouble: Feminism and the subversion of identity GT. Political Theory, 4, 4-24.

Cáceres, C. F., Salazar, X., Rosasco, A. M. y Fernández Dávila, P. (2002). Ser un hombre en el Perú de hoy. Una mirada a la salud sexual desde la infidelidad, la violencia y la homofobia. Redess Jóvenes.

Cappi Manzini, L. y do Horto Salles Tiellet, M. (2014). Profesoras y docencia de estudiantes del sexo masculino de práctica profesional en una guardería: Desafíos en las relaciones entre géneros. Subjetividad y Procesos Cognitivos, 18(2), 71-97. https://www.redalyc.org/ pdf/3396/339634157004.pdf

Clyde, M. (1989). Who is the odd man out: Men in early childhood settings? Early Child Development and Care, 52(1-4), 93-99. https://doi.org/10.1080/0300443890520107

Connell, R. W. (2005). Masculinities (2. ${ }^{a}$ ed.). Polity.

Corbin, J. M. y Strauss, A. (1990). Grounded theory research: Procedures, canons, and evaluative criteria. Qualitative Sociology, 13(1), 3-21. https://doi.org/10.1007/BF00988593

Creswell, J. W. y Poth, C. N. (2018). Qualitative inquiry y research design. Sage Publications.

Cunningham, B. y Watson, L. W. (2002). Recruiting male teacher. Young Children 57(6), 10-15. http://archivio.maschiselvatici.it/semplicistrategie.pdf

Davies, B. (1989). The discursive production of the male/female dualism in school settings. Oxford Review of Education, 15(3), 229-241. https://doi.org/10.1080/0305498890150304

Eagly, A. H. y Mladinic, A. (1989). Gender stereotypes and attitudes toward women and men. Personality and Social Psychology Bulletin, 15(4), 543-558. https://doi. org/10.1177/0146167289154008

Faur, E. (2004). Masculinidades y desarrollo social. Las relaciones de género desde la perspectiva de los hombres. Arango Editores. https://issuu.com/fundacionjyg/docs/masculinidades y desarrollo social 
http://doi.org/10.15359/ree.25-3.33

http://www.una.ac.cr/educare

educare@una.ac.cr

García Villanueva, J. y Hernández Ramírez, C. I. (2015). La formación del profesorado de nivel preescolar: Un campo que excluye a los hombres en la ciudad de México. Entreciencias: Diálogos en la Sociedad del Conocimiento, 3(7), 241-251. https://doi.org/10.21933/J. EDSC.2015.07.135

Haslanger, S. (2012). Resisting reality. Social construction and social critique. Oxford University Press. https://doi.org/10.1093/acprof:oso/9780199892631.001.0001

Instituto Nacional de Estadística e Informática. (2017). Principales resultados de la encuesta nacional a instituciones educativas de nivel inicial, primaria y secundaria 2016. https://www. inei.gob.pe/media/MenuRecursivo/publicaciones digitales/Est/Lib1466/

Joseph, S. y Wright, Z. (2016). Men as early childhood educators: Experiences and perspectives of two male prospective teachers. Journal of Education and Human Development, 5(1), 213219. https://doi.org/10.15640/jehd.v5n1a22

Lomazzi, V., Israel, S. y Crespi, I. (2019). Gender equality in Europe and the effect of workfamily balance policies on gender-role attitudes. Social Sciences, 8(1), 1-29. https://doi. org/10.3390/socsci8010005

Lorber, J. (1994). "Night to his day": The social construction of gender. En Paradoxes of gener (pp. 5465). Yale University Press. http://seas3.elte.hu/coursematerial/GallCecilia/Lorber Night to his day.pdf

Muñoz Restrepo, D. y Vásquez Holguín, X. (2017). Pasantía en Chile: Una ampliación de saberes y conocimientos sobre la educación infantil. Journal of students, (2), 46-55. https://doi. org/10.21501/25007858.2578

Osses Bustingorry, S., Sánchez Tapia, I. e Ibañez Mansilla, F. M. (2006). Investigación cualitativa en educación. Hacia la generación de teoría a través del proceso analítico. Estudios Pedagógicos, 32(1), 119-133. https://doi.org/10.4067/S0718-07052006000100007

Páramo Morales, D. (2015). La teoría fundamentada (Grounded Theory), metodología cualitativa de investigación científica. Pensamiento \& Gestión, (39), 7-13. https://www.redalyc.org/ pdf/646/64644480001.pdf

Pardo, M. y Adlerstein, C. (Consultoras). ((2015). Informe nacional sobre docentes para la educación de la primera infancia: CHILE. UNESCO-OREALC. http://ceppe.uc.cl/images/contenido/ publicaciones/proyecto-estrategico-regional/2012/Informe-Primera-Infancia-CHILE.pdf

Pico Villancís, D. A. (2017). La formación del docente masculino en la educación preescolar [Tesis de licenciatura]. Universidad Técnica de Ambato. https://repositorio.uta.edu.ec/ handle/123456789/26363 
http://doi.org/10.15359/ree.25-3.33

Pizarro Quiñones, A. (2018). Una aproximación funcional al problema de la representación en la teoría feminista [Tesis de licenciatura]. Pontificia Universidad Católica del Perú. http:// repositorio.pucp.edu.pe/index/handle/123456789/151964

Qu, S. Q. y Dumay, J. (2011). The qualitative research interview. Qualitative Research in Accounting \& Management 8(3), 238-264. https://doi.org/10.1108/11766091111162070

Sargent, P. (2005). The gendering of men in early childhood education. Sex Roles, 52(3-4), 251259. https://doi.org/10.1007/s11199-005-1300-x

Sánchez Barrenechea, J. (2011). Percepciones sobre el trabajo extradoméstico de mujeres empresarias de Gamarra [Tesis de maestría]. Pontificia Universidad Católica del Perú. http:// tesis.pucp.edu.pe/repositorio/handle/20.500.12404/1403

Valdivia Santa Cruz, S. R. (2013). ¿Mamá ya estás viniendo? Varones y mujeres proveedores de recursos y cuidados [Tesis de maestría]. Pontificia Universidad Católica del Perú. http:// tesis.pucp.edu.pe/repositorio/handle/20.500.12404/5216

Vendrell, R., Dalmau, M., Gallego, S. y Baqués, M. (2015). Los varones, profesional en educación infantil. Implicaciones en el equipo pedagógico y en las familias. Revista de Investigación Educativa, 33(1), 195-210. https://doi.org/10.6018/rie.33.1.184051

West, C. y Zimmerman, D. (1987). Doing Gender. Gender \& Society, 1(2), 125-151. https://doi. org/10.1177/0891243287001002002

Wood, G. (2008). Gender stereotypical attitudes: Past, present and future influences on women's career advancement. Equal Opportunities International, 27(7), 613-628. https://doi. org/10.1108/02610150810904319

Zhang, W. (2017) Male teachers in early childhood education: Why more men? A Review of the Literature [Tesis de maestría]. St. Cloud State University. https://repository.stcloudstate. edu/cgi/viewcontent.cgi?article $=1022 \&$ context $=$ cfs etds 